\title{
Beitrag zur Messung von Fliessgeschwindigkeiten in Klärbecken mit Thermosonden
}

\author{
Von L. KaLMaN \\ Eidg. Anstalt für Wasserversorgung, Abwasserreinigung und Gewässerschutz (EAWAG) an der \\ Eidg. Technischen Hochschule, Zürich, Direktion: Prof. Dr. O. JAAG
}

Manuskript eingegangen am 14. Dezember 1965

\section{A. Entwicklung einer Thermosonde für Geschwindigkeitsmessungen}

\section{Vorbemerkungen}

Die Entwicklung der Geschwindigkeitsmessung in strömenden Medien mit Hilfe des thermischen Abkühlungsprinzips wurde schon frühzeitig in Angriff genommen. Den Vorschlag, mit elektrisch geheizten Widerständen Geschwindigkeiten zu messen, findet man schon um 1913 bei H. Gerdien. Die Theorie des Hitzdrahtanemometers entwickelten BaIley und Simmons um 1927. Dieses Prinzip hat den Vorteil, dass es auch bei kleinen Geschwindigkeiten angewendet werden kann, wenn bereits Messmethoden mit Flügel oder Staurohr versagen. Von den heute bekannten Instrumenten sei das «Fierz»-Anemometer für Luft erwähnt, mit dem man Luftgeschwindigkeiten von $5 \mathrm{~cm} / \mathrm{sec}$ an aufwärts messen kann. Dieses verwendet ein sogenanntes Thermokreuz, bestehend aus einem geheizten Widerstand mit angelötetem Thermoelement.

Bei Flüssigkeitsmessungen sind die Probleme schwieriger. Es sind auch auf diesem Gebiet einige Geräte entwickelt worden, die aber nur Einzelausführungen sind. So beschreibt WÄHR ein Instrument mit zwei Hitzdrähten, wobei der Widerstand beider Drähte verglichen wird [1].

Ein bereits in der Praxis verwendetes Instrument ist die vom Max-Planck-Institut in Göttingen entwickelte Thermosonde, welche bereits für Messungen in Absetzbecken eingesetzt wurde. Der Messkopf ist eine geheizte Sonde von $4 \mathrm{~mm}$ Durchmesser und $30 \mathrm{~mm}$ Länge. Die Temperatur wird mit einem Thermoelement gemessen, wobei die Vergleichslötstelle ebenfalls im Wasser liegt, wodurch eine Temperaturkompensation erreicht wird. Die kleinste noch messbare Geschwindigkeit beträgt etwa $5 \mathrm{~mm} / \mathrm{sec}$. Der Thermoelemente wegen musste ein hochempfindliches Spiegelgalvanometer verwendet werden, welches bei Transport und Aufstellung sehr störanfällig ist. Ausserdem ist keine Möglichkeit zur Abdeckung der Sonde vorhanden, wodurch die Einstellung der Geschwindigkeit auf Null schwierig sein muss. 
Über den Aufbau des Gerätes orientiert ein Aufsatz von WACK [2]. Ủber den praktischen Einsatz für die Strömungsmessungen in einem Absetzbecken berichtet Schmidt-Bregas [3]. Dieser Publikation ist u. a. zu entnehmen, dass der Einfluss der Temperatur vernachlässigt werden kann. Ebenfalls zeigten sich bei Reinwasser nur unwesentliche Unterschiede gegenüber Abwasser.

\section{Das Strömungsmessgerät der EAWAG}

Das Strömungsmessgerät der EAWAG verwendet als Mess- und Kompensationselemente Thermistoren. Diese Halbleiter haben einen sehr starken negativen Temperaturkoeffizienten, weshalb sie auch als NTC-Widerstände bezeichnet werden. Solche Thermistoren werden z.B. von der Firma Philips als in Glas eingeschlossene Präzisions- und Miniaturausführungen hergestellt und sind in den elektrischen Eigenschaften sehr beständig. Die Anwendung der Thermistoren hat gegenüber der Verwendung von Thermoelementen folgende Vorteile:

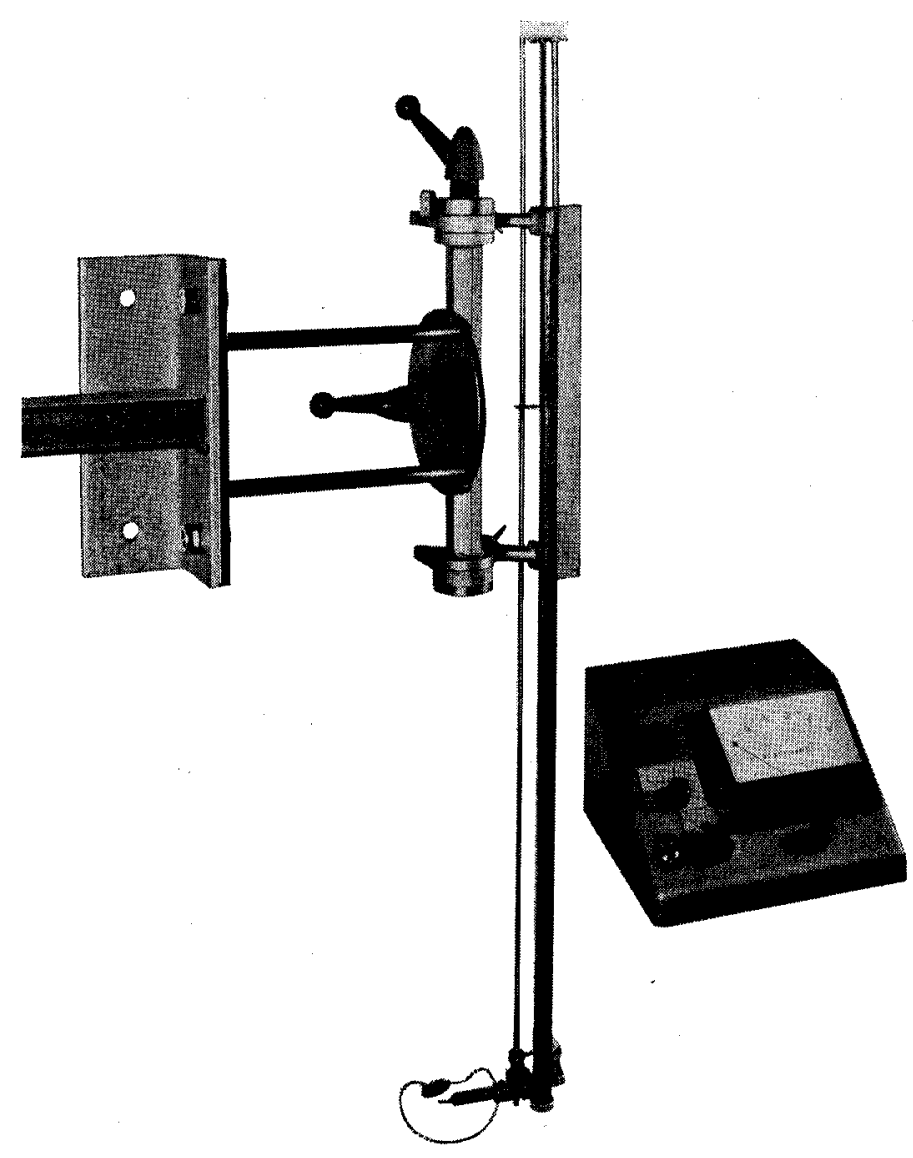

Abbildung 1

Ansicht des Instruments. 
1. Einfacherer Aufbau der Schaltung und des Messgerätes, da keine separate Heizung des Messkopfes; ebenso ist auch kein Verstärker, bzw. kein hochempfindliches Galvanometer notwendig.

2. Abmessung und Erwärmung der Meßspitze sehr klein, dadurch nur geringe Störung der Strömung.

3. Grössere Messempfindlichkeit für kleine Geschwindigkeiten.

4. Kleinerer Stromverbrauch, daher Betrieb mit kleinen Batterien möglich.

Diesen Vorteilen stehen folgende Nachteile gegenüber:

1. Meßspitze aus Glas, daher zerbrechlich. Sofern mit der Drosselkappe gemessen wird, ist der Thermistor gut geschützt; ohne Drosselkappe ist ein besonderer Schutz notwendig.

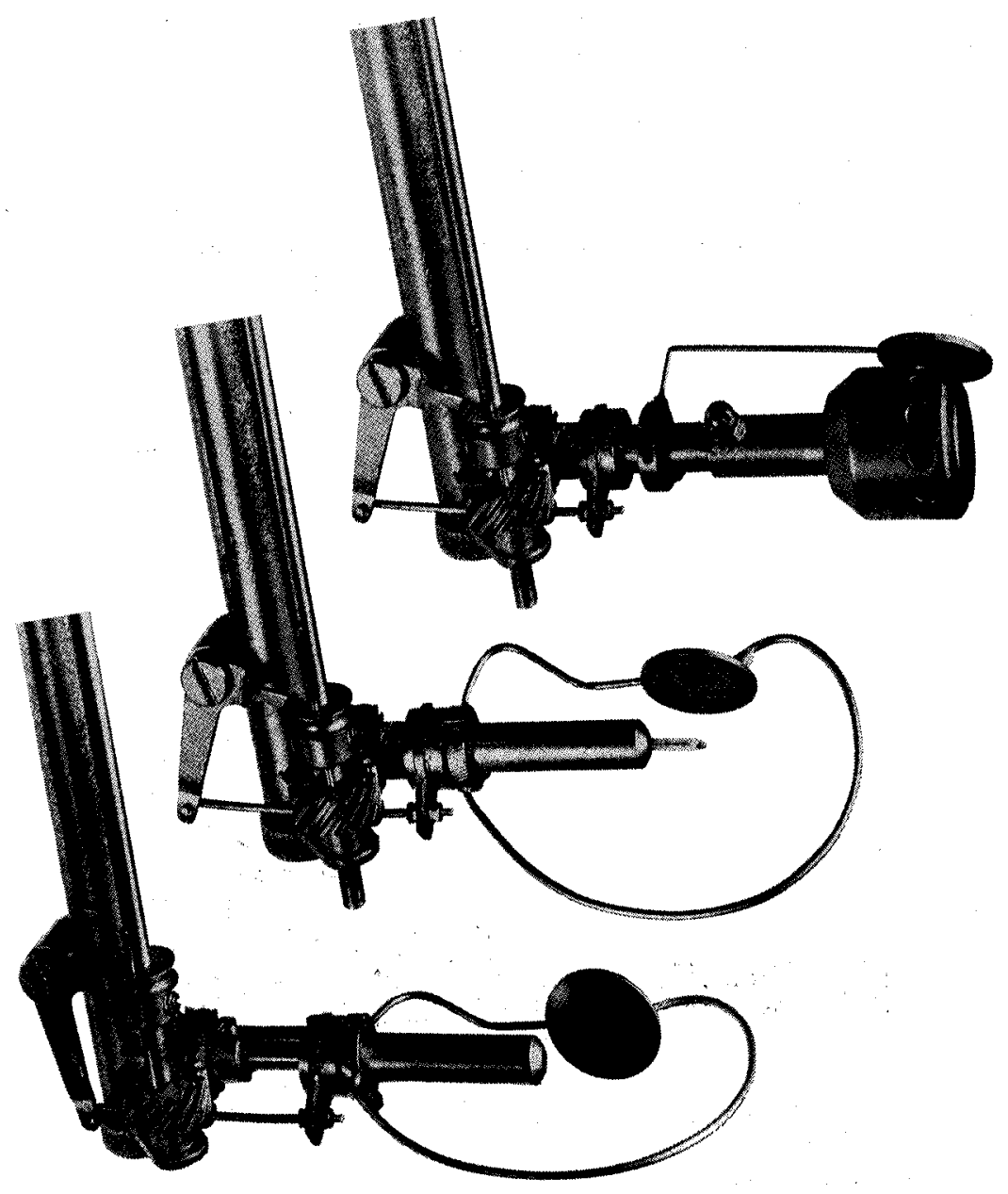

Abbildung 2

Detailansicht des Instruments. 
2. Die Empfindlichkeit ist temperaturabhängig, daher muss bei der Auswertung entweder die jeweilige Temperatur berücksichtigt oder eine Linearisierungsschaltung vorgesehen werden.

3. Wegen der kleinen Abmessungen der MeBspitze auf Luftblasenbildung und Verschmutzung empfindlicher.

\section{Aufbau des Messgerätes}

Der schematische Aufbau des Messgerätes ist aus Abb. 3 ersichtlich, die elektrische Schaltung aus Abb. 4. Der Thermistor ist in einem rostfreien Stahlrohr von $5 \mathrm{~mm}$ Durchmesser mit Araldit eingegossen. Die Spitze des Thermistors von $2 \mathrm{~mm}$ Durchmesser steht etwa $10 \mathrm{~mm}$ vor. Der Thermistor befindet sich in einer konzentrischen Schiebekappe, mit welcher der Thermistor abgedeckt werden kann. Auf diese Weise wird ein geschwindigkeitsloser Zustand erzeugt, damit die Nullpunktkorrektur vorgenommen werden kann. Diese Verschiebung der Kappe kann mit einem Mechanismus von oben betätigt werden.

Allerdings entspricht dieser Zustand in der Kappe nicht vollständig der ruhenden Flüssigkeit, da die Verhältnisse der Wärmeabgabe dort etwas anders sind. Da diese Differenz jedoch durch Messung feststellbar, ist, kann sie in den Eichkurven berücksichtigt werden. Die Nullpunktkorrektur wird mit einem Potentiometer $R_{\mathbf{2}}$ in der Brücke vorgenommen. Die Stellung des Potentiometers ist bei richtiger Kompensation unabhängig von der Temperatur. Anderseits ist sie jedoch von der Art des Wassers

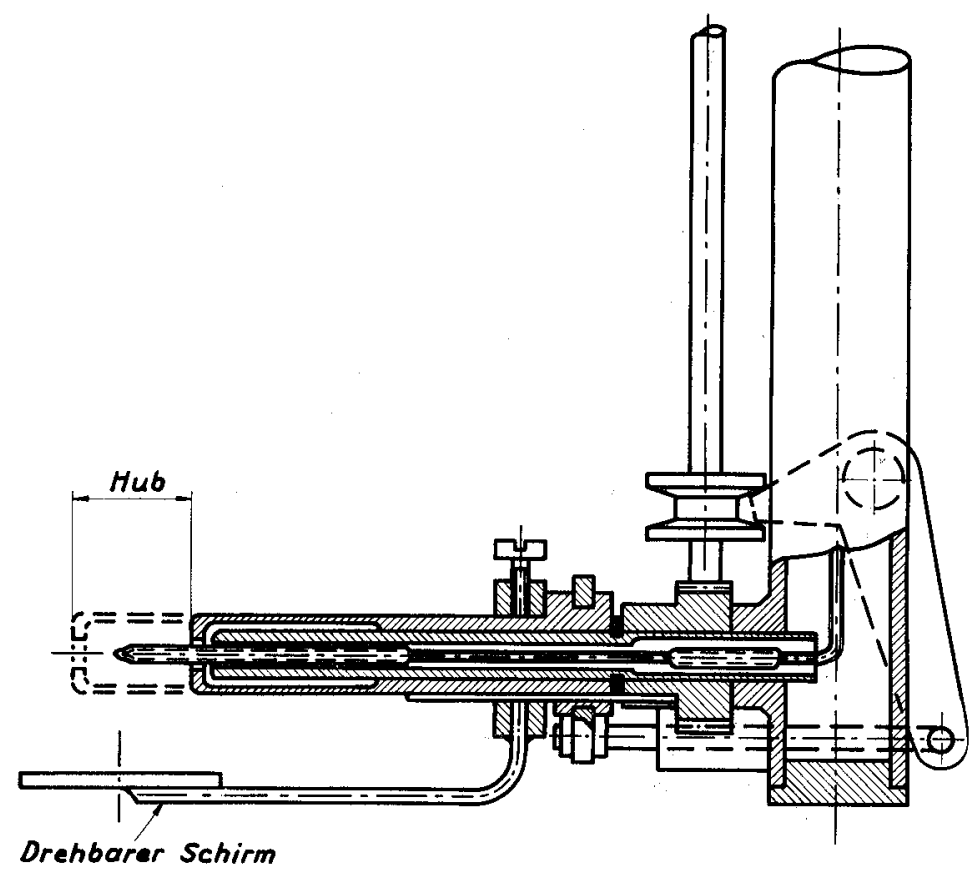

Abbildung 3

Schematische Zeichnung des Instruments. 


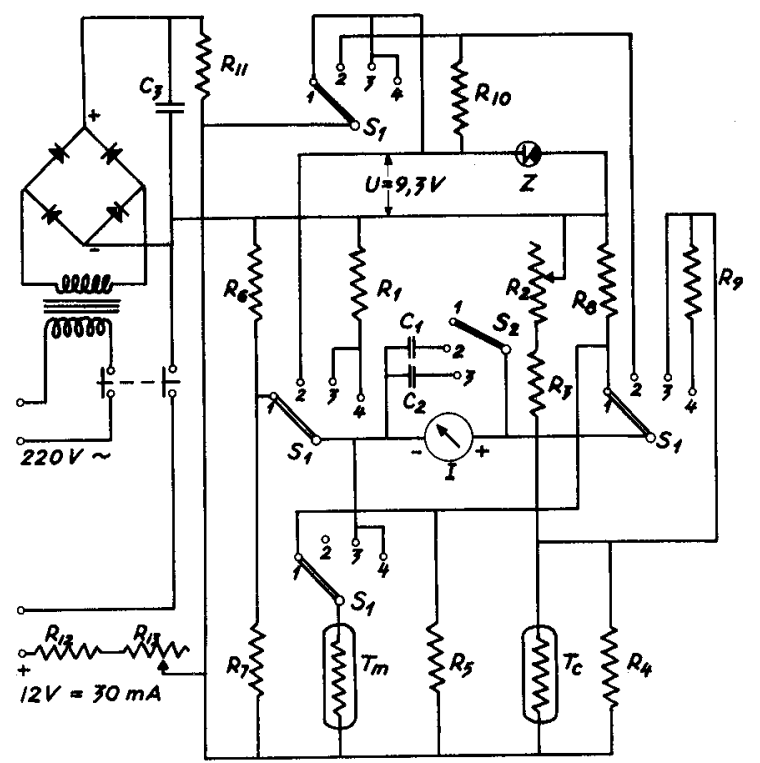

$R_{2}$ Nullpunktkorrektur der Geschwindigkeitsmessung

$R_{13}$ Batteriekontrolle

$S_{1}$ Stufenschalter

Stellung 1: Temperaturmessung

Stellung 2 : Batteriekontrolle

Stellung 3: Geschwindigkeitsmessbereich 1

Stellung 4: Geschwindigkeitsmessbereich 2

$S_{2}$ Stufenschalter

Stellung 1: ohne Dämpfung

Stellung 2: Dämpfung $T=10 \mathrm{sec}$

Stellung 3: Dämpfung $T=40 \mathrm{sec}$

Widerstände:
$R_{1}=305 \Omega$
$R_{2}=1000 \Omega$
$R_{3}=2200 \Omega$
$R_{4}=33000 \Omega$
$R_{5}=510 \Omega$
$R_{6}=10820 \Omega$
$R_{7}=390 \Omega$
$R_{8}=11800 \Omega$
$R_{9}=5700 \Omega$
$R_{10}=51,8 \Omega$
$R_{11}=500 \Omega$
$R_{12}=50 \Omega$
$T_{m}=$ Messthermistor Philips E $205 \mathrm{CE} / \mathrm{P} 1 \mathrm{~K}: 1000 \Omega / 20^{\circ} \mathrm{C}$
$T^{m}=$ Kompensationsthermistor Philips B8 $32003 \mathrm{P} 6 \mathrm{~K} 8: 6800 \Omega / 20^{\circ} \mathrm{C}$
$Z^{c}=$ Zenerdiode Philips BZZ $209,3 \mathrm{~V} / 30 \mathrm{~mA}$
$I=$ Messinstrument $50 \mu \mathrm{A} / 1800 \Omega$

$R_{13}=$

$150 \Omega$

Abbildung 4

Schaltschema mit Stückliste.

abhängig, nämlich von dessen Salzgehalt, Feststoffgehalt u.a.m. Diese Stellung ist auch ein Mass für die Grösse der Wärmeübergangszahl an der Thermistorspitze. Sofern diese Zahl nicht stark verschieden ist von derjenigen für reines Wasser, kann man auch die Eichkurven für reines Wasser mit genügender Genauigkeit verwenden.

\section{Bestimmung der Strömungsgeschwindigkeit}

Nachdem die Nullpunktkorrektur vorgenommen wurde, wird bei zurückgezogener Schiebekappe die Geschwindigkeit mit einem passend gewählten Messbereich be- 
stimmt. Sofern die Geschwindigkeit zu gross ist, kann der Messbereich mit Hilfe der entsprechenden Drosselkappe nach oben, erweitert werden. Die Drosselkappe ist so ausgebildet, dass an der Meßspitze nur ein kleiner Teil des Wassers vorbeifliesst. Die Drosselwirkung entsteht aber durch Teilung des Wasserstroms und nicht durch Drosselung. Deshalb sind auch diese Drosselkappen im Abwasser weniger Verstopfungen ausgesetzt. Kappen mit Filtersieben usw. eignen sich aus diesem Grunde nicht.

Sobald die Geschwindigkeit turbulent ist, tritt auch bei stationärer Strömung eine pulsierende Geschwindigkeitsanzeige auf. Flügelinstrumente haben wegen ihrer rotierenden Masse und dem grossen erfassten Strömungsquerschnitt einen genügend guten Ausgleich. Da aber die Zeitkonstante des Thermistors nur etwa $2 \mathrm{sec}$ beträgt, folgt die Anzeige dem Augenblickswert der Geschwindigkeit. Um trotzdem eine Ablesung des Mittelwertes zu erhalten, wurden bei diesem Instrument Dämpfungsglieder in Form von zuschaltbaren Kondensatoren vorgesehen, die eine bequeme Ablesung auch bei stark schwankender Wasserströmung erlauben.

Die am Instrument ablesbare Geschwindigkeit ist die mittlere Geschwindigkeit $\bar{v}=\int v d t / \int d t$.

Die Zeitkonstante der Dämpfung beträgt $T=C R$, wobei

$T=$ Zeitkonstante der Dämpfung in Sekunden,

$C=$ Kapazität des Kondensators in Farad,

$R=$ Widerstand des Galvanometers in $\Omega$.

Dadurch ergibt sich mit $R=2000 \Omega, C=5000$ bzw. $20000 \mu \mathrm{F}, T=10$ bzw. 40 sec.

\section{Bestimmung der Strömungsrichtung}

Vor der Thermistorspitze kann in einem Abstand von etwa $1,5 \mathrm{~cm}$ ein beweglicher, runder Schirm angebracht werden. Sobald der Thermistor in die Totwasserzone des Schirms kommt, zeigt sich ein Minimum in der Anzeige der Geschwindigkeit, wodurch deren Richtung bestimmt werden kann. Die Betätigung der Drehung um die horizontale Achse erfolgt mit Hilfe eines Winkeltriebs mit der gleichen senkrechten Welle, mit der auch die Schiebekappe betätigt wird. Die Drehung um die senkrechte Achse erfolgt durch Lagerung in der Einspannvorrichtung. Beide Winkelstellungen können von oben abgelesen werden.

Die Bestimmung der Strömungsrichtung mit dem minimalen Ausschlag ist um so ausgeprägter, je kleiner die Reynoldssche Zahl ist. Bei kleiner Geschwindigkeit kann das Minimum sogar zu Null werden.

\section{Empfindlichkeit des Instruments}

\begin{tabular}{lrr}
\hline Messbereich & \multicolumn{1}{l}{1} & \multicolumn{1}{l}{2} \\
\hline Ohne Drosselkappe & $2-8 \mathrm{~mm} / \mathrm{s}$ & $2-16 \mathrm{~mm} / \mathrm{s}$ \\
Mit Drosselkappe 1 & $15-60 \mathrm{~mm} / \mathrm{s}$ & $15-300 \mathrm{~mm} / \mathrm{s}$ \\
Mit Drosselkappe 2 & \multicolumn{1}{c}{$-150-500 \mathrm{~mm} / \mathrm{s}$} \\
\hline
\end{tabular}

\section{Luftblasenbildung}

Eine unangenehme Erscheinung ist das Auftreten von kleinen Luftbläschen an der Thermistorspitze, die die Messung beeinträchtigen. Diese Blasenbildung stellt sich bei allen geheizten Sonden ein, die in nahezu luftgesättigtes Wasser getaucht werden. 
Allerdings gibt es zwei Möglichkeiten, um sich gegen diese Luftblasen zu schützen, welche die Anwendung von Thermosonden im Wasser allgemein erschweren.

Die erste Massnahme besteht darin, dass man den Heizstrom so knapp als zulässig bemisst; anderseits kann durch die Verwendung von Netzmitteln, die die Oberflächenspannung herabsetzen, das Haften von Bläschen verhindert werden. Gut bewährt hat sich Petrol, doch hört dessen Wirkung nach etwa 5-6 Stunden auf.

\section{Verschmutzung des Thermistors}

Ein anderer störender Einfluss rührt von den Schwebestoffen her, die sich am Thermistor ablagern, wodurch der Wärmeübergang verändert und dadurch der Nullpunkt verschoben wird. Die vorgeschobene Schiebekappe bietet daher den Vorteil, das Auftreten einer Verschmutzung feststellen zu können. Anderseits kann durch einfaches Vor- und Rückziehen der Schiebekappe mit einem durch Pumpenkolbenwirkung erzeugten Wasserstrahl die Thermistorspitze von den abgelagerten Flocken gereinigt werden, ohne dass das Instrument aus der Meßstellung ausgebaut werden muss. Durch einen in der Schiebekappe angebrachten Gummiring kann die Reinigungswirkung noch verstärkt werden.

\section{Temperaturmessung}

Durch eine Umstellung des Messbereichumschalters wird das Instrument in eine temperaturanzeigende Messbrücke umgewandelt. Die Strombelastung des Thermistors ist in diesem Falle nur sehr gering, um keine Eigenerwärmung hervorzurufen. Die gleichzeitige Feststellung der Temperatur in verschiedenen Tiefen auf bequeme Art ist sehr nützlich, unter anderem dann, wenn durch Temperaturdifferenzen in einem Becken Strömungen entstehen oder beeinflusst werden. Weil die Wärmeträgheit sehr klein ist, braucht man, im Gegensatz zu einem Quecksilberthermometer, bei der Messung nicht zu warten.

Da weder der Thermistor noch die Brücke bei so grossen Widerstandsänderungen eine lineare Charakteristik haben, müsste die Temperaturablesung mit Hilfe eines Diagramms oder mit einer separaten, geeichten Skala erfolgen. Um dies zu vermeiden, ist mit dem Messthermistor bei der Temperaturmessung ein Widerstand $R_{\mathbf{5}}$ parallel geschaltet, wobei durch dessen passende Wahl eine ausreichende Linearität in einem Bereich von $0-30^{\circ} \mathrm{C}$ erreicht werden kann. Damit ist es möglich, die Temperatur direkt in Graden abzulesen.

\section{Stromversorgung}

Sofern kein Netz zur Verfügung steht, kann das Gerät auch mit einer Batterie von $12 \mathrm{~V}$ mit einem Stromverbrauch von etwa $30 \mathrm{~mA}$ betrieben werden. Da die Brückenspannung durch Zenerdioden sehr gut stabilisiert ist, spielt ein Nachlassen der Batteriespannung um einige Volt keine Rolle.

\section{Einfluss der Wassertemperatur}

Wie wir noch später sehen werden, verursacht die maximale messbare Geschwindigkeit nur eine Abkühlung von etwa $3^{\circ} \mathrm{C}$, so dass das Instrument sehr temperatur- 
empfindlich sein muss, um bei kleinen Fliessgeschwindigkeiten noch messen zu können. Es ist daher sehr wichtig, dass der Einfluss der jeweiligen Wassertemperatur, die eine Verschiebung des Nullpunktes und eine Änderung der Empfindlichkeit verursachen würde, ausgeschaltet wird.

\subsection{Kompensation der Flüssigkeitstemperatur für den Nullpunkt}

Zwei Thermistoren befinden sich in je einem der Brückenzweige und sollen so eine Temperaturkompensation ermöglichen. Da der Messthermistor vom Wasser bespült, der Kompensationsthermistor dagegen in einer festen Masse im unteren Rohrende eingegossen ist, muss der erste ein niederohmiger, der zweite ein hochohmiger Typ sein.

Die Temperaturempfindlichkeit der beiden Thermistoren ist verschieden, weshalb eine Kompensation nicht ohne weiteres gewährleistet ist. Sofern die Temperaturempfindlichkeit des Kompensationsthermistors grösser ist als die des Messthermistors, ist eine Kompensation möglich, indem die Wirkung des Kompensationsthermistors mit einem $\mathrm{zu}$ ihm parallelen Widerstand $R_{4}$ verkleinert wird. Die Abstimmung wird so vorgenommen, dass abwechselnd das Instrument in ruhendes kaltes und warmes Wasser eingetaucht und der Widerstand $R_{4}$ so lange geändert wird, bis man eine volle Kompensation für den Nullpunkt erreicht.

11.2 Änderung der Empfindlichkeit mit der Temperatur und deren Kompensierung

Die Empfindlichkeit des Thermistors ist keine lineare Funktion der Temperatur, und auch die Joulesche Wärmeabgabe des Thermistors ist temperaturabhängig. Daher muss man entweder bei der Auswertung die Flüssigkeitstemperatur berücksichtigen oder eine Kompensierung vorsehen. Die Kompensierung kann durch passende Wahl der Widerstände in der Brücke erreicht werden.

Die in der Zeiteinheit abgegebene Wärmemenge $Q$ an der Thermistorspitze kann auf Grund der Wärmedurchgangsgleichung folgendermassen ermittelt werden:

$$
\frac{t_{m t h}-t_{w}}{Q}=\frac{l}{\alpha F}+\frac{s}{\lambda F_{m}}, \text { wobei }
$$

$Q \quad=$ Wärmeabgabe des Messthermistors,

$t_{m t h} \quad=$ Temperatur im Innern des Messthermistors,

$t_{w} \quad=$ Wassertemperatur,

$F \quad=$ Thermistoroberfläche,

$\alpha \quad=$ Wärmeübergangszahl an der Thermistoroberfläche,

$\lambda=$ Wärmeleitzahl der Thermistorwand,

$F_{m}$ und $s=$ Fläche bzw. Wandstärke des Thermistors.

Aus dieser Gleichung ist ersichtlich, dass, um hohe Empfindlichkeit zu erreichen, die Thermistoroberfläche möglichst klein sein soll im Verhältnis zur Wärmeabgabe des Thermistors.

Von der Wärmeübergangszahl $\alpha$ ist bekannt, dass sie im ruhenden Wasser von der Grashofschen Zahl und somit stark von der Temperaturdifferenz $t_{m i h}-t_{w}$ abhängig ist. Bei bewegtem Wasser dagegen hängt sie von der Reynoldsschen und von der Prandtl- und Nusselt-Zahl ab. Durch deren Beziehung lässt sich ermitteln, dass die Wärmeübergangszahl in dem uns interessierenden Temperaturbereich nur von der Geschwindigkeit und nicht von der Wassertemperatur abhängig ist. Somit kann man für 


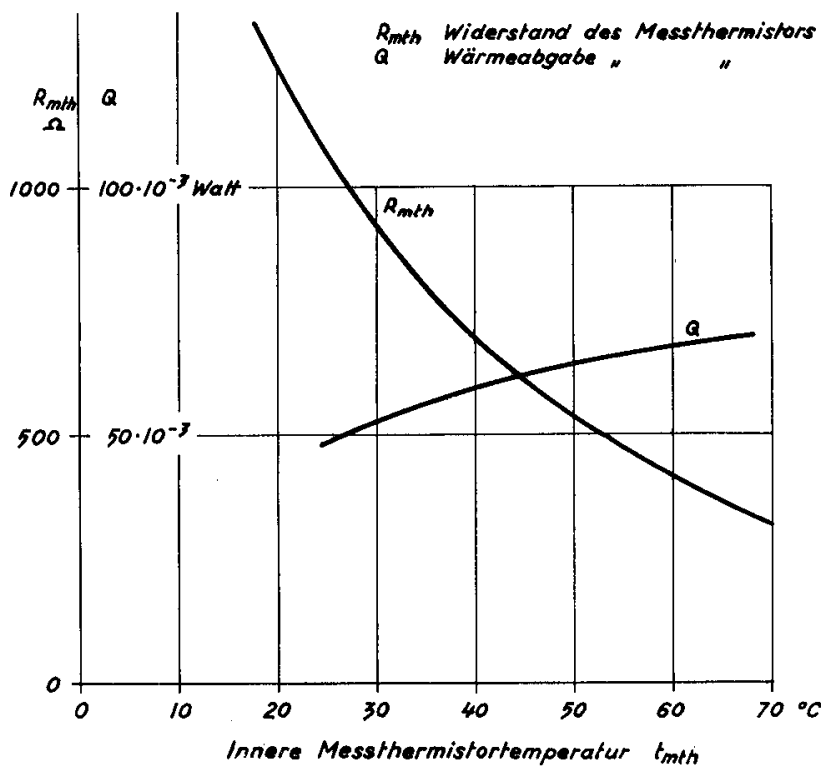

Abbildung 5

Charakteristik des Messthermistors.

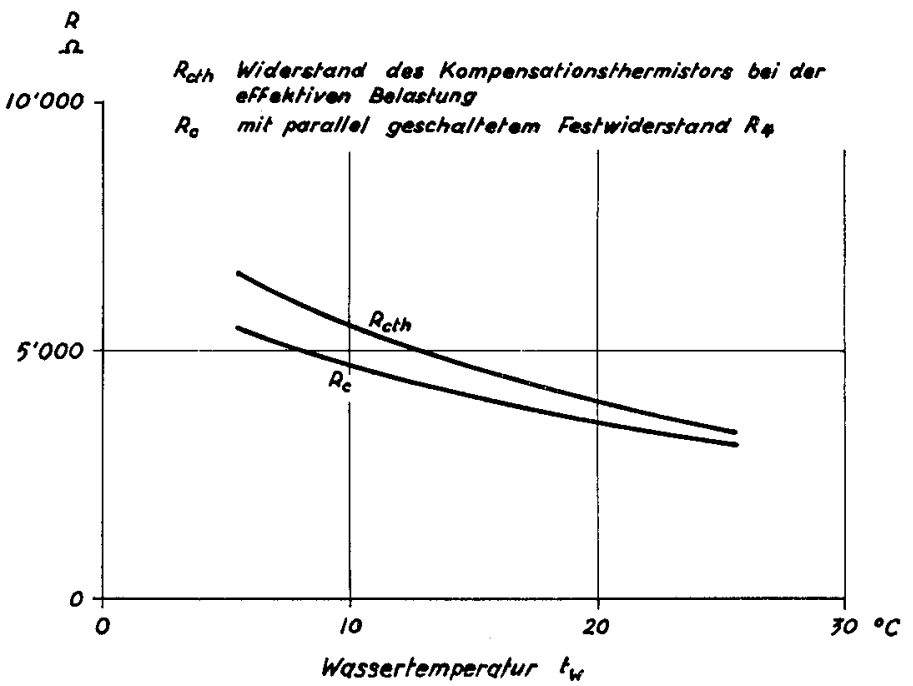

Abbildung 6

Charakteristik des Kompensationsthermistors. 
den angeströmten Thermistor folgern, dass die Grösse $\frac{t_{m l h}-t_{w}}{Q}$ als eine nur von der Wassergeschwindigkeit abhängige Funktion angesehen werden kann. Anderseits ist die Wärmeabgabe $Q$ des Thermistors eine Funktion des Widerstandes $R_{m t h}$ des Messthermistors, der wiederum nur von der Temperatur $t_{m t h}$ abhängig ist.

$$
Q=i_{m t h}^{2} R_{m t h} \text {. }
$$

Um zu prüfen, wie ein bei einer bestimmten Wassertemperatur geeichtes Thermistorinstrument sich bei anderen Wassertemperaturen verhält, geht man am besten rechnerisch vor.

Zunächst wird durch Messung der Widerstand $R_{m t h}$ und daraus die Wärmeabgabe $Q$ des Messthermistors in Funktion der Temperatur $t_{m t h}$ bestimmt (Abb. 5).

Anderseits kann man den Widerstand $R_{c t h}$ des Kompensationsthermistors bzw. $R_{c}$ mit dem parallelgeschalteten, festen Widerstand $R_{\mathbf{4}}$ zusammen in Funktion der Wassertemperatur $t_{w}$ bei normaler Strombelastung mit dem ins Wasser getauchten Instrument durch Messung bestimmen (Abb. 6).

Aus diesen nun bekannten Widerstandskurven lässt sich bei gegebenem Widerstand $R_{g}$ des Mikroamperemeters der Instrumentenstrom $i$ in Funktion der Wassertemperatur $t_{w}$ und der inneren Messthermistortemperatur $t_{m t h}$ berechnen. Diese Berechnung erfolgt mit Hilfe der bekannten Gleichung für den Brückenstrom, die in diesem Fall folgende Form hat:

$i=U \frac{R_{m t h}\left(R_{2}+R_{3}\right)-R_{1} R_{c}}{R_{g}\left(R_{m t h}+R_{1}\right)\left(R_{c}+R_{2}+R_{3}\right)+R_{m t h} R_{1}\left(R_{c}+R_{2}+R_{3}\right)+R_{c}\left(R_{2}+R_{3}\right)} \overline{\left(R_{m t h}+R_{1}\right)}$.

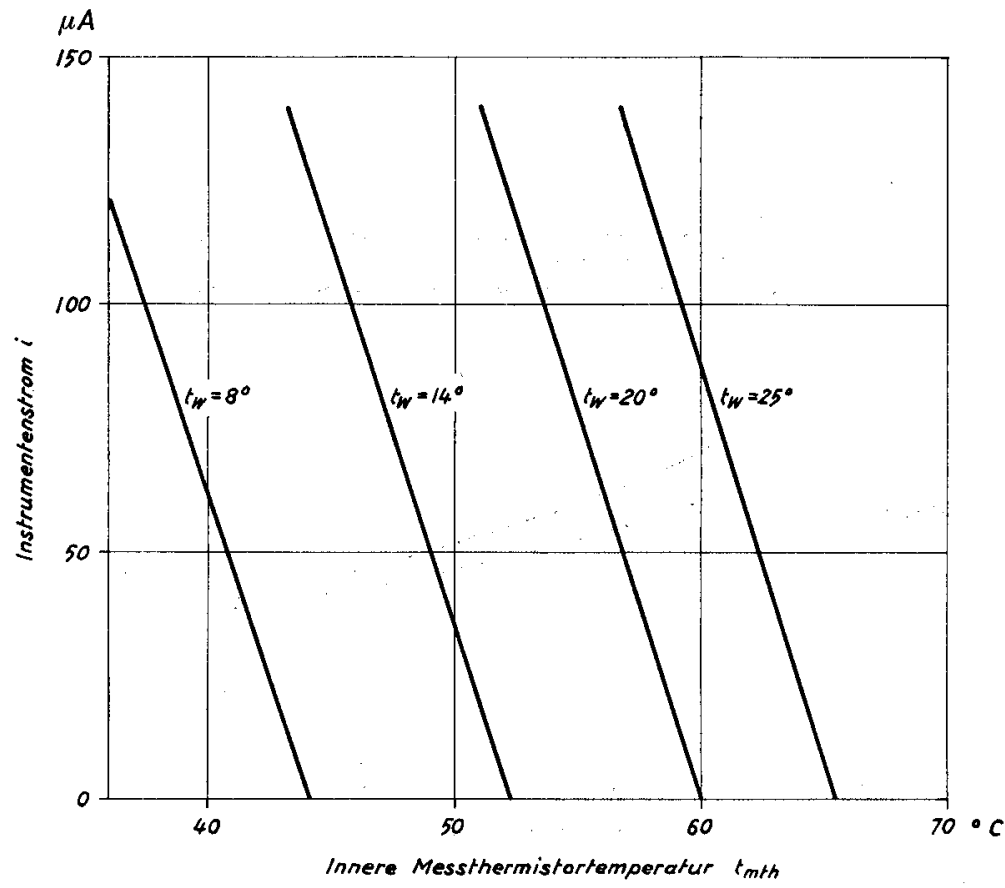

Abbildung 7

Charakteristik des Instrumentenstroms. 


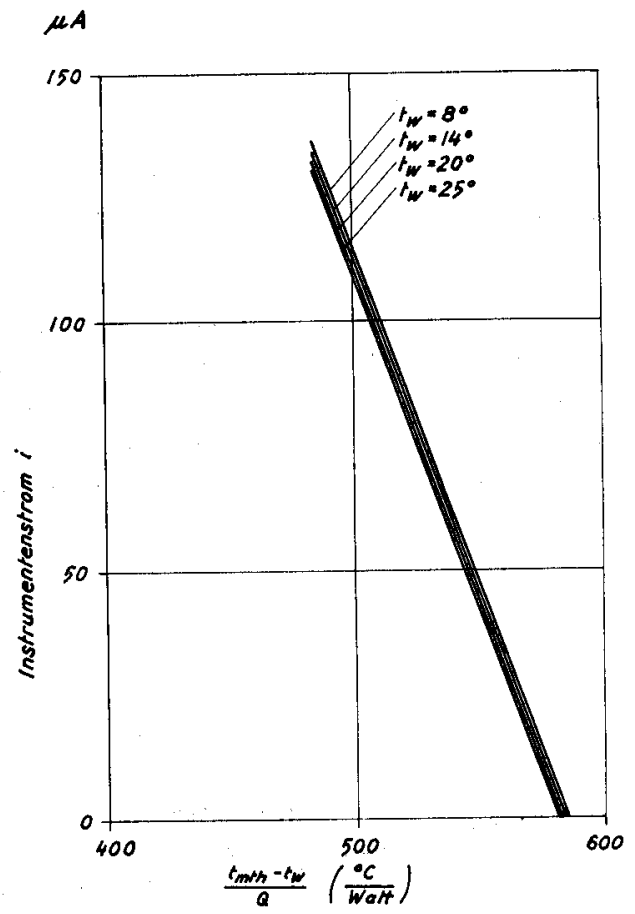

Abbildung 8. Instrumentenstrom und Wassertemperatur.

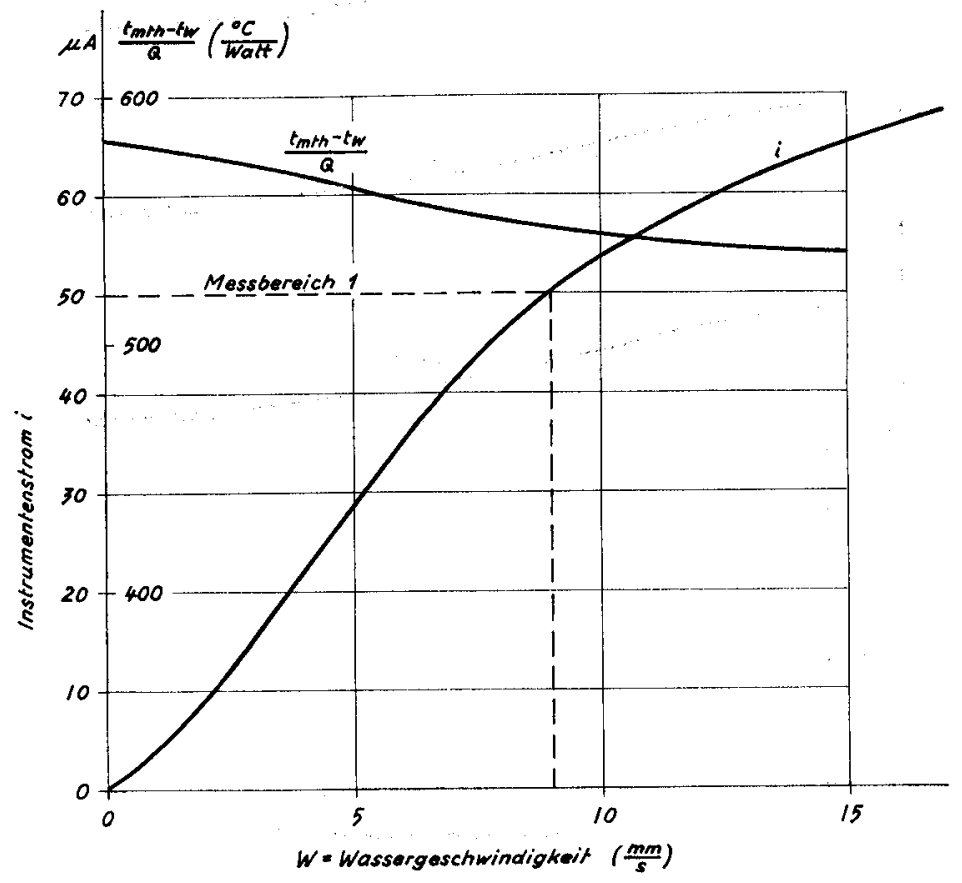

Abbildung 9. Eichkurve. 
Anderseits kann aus den Funktionen der Abb. 5 und 7 die Kurvenschar für den Instrumentenstrom $i$ ermittelt werden, in Abhängigkeit von der Grösse $\frac{t_{m t h}-t_{w}}{Q}$, die nur von der Wassergeschwindigkeit abhängig ist. Der Parameter ist dabei die Wassertemperatur $t_{w}$ (Abb. 8).

Wird die Kompensation richtig gewählt, so müssen diese Linien möglichst zusammenfallen, wie es in Abb. 8 ausreichend der Fall ist. Sind die Linien nur parallel verschoben, so kann der Fehler durch die erwähnte Nullpunktkompensation berichtigt werden. Würde dagegen ihre Neigung, und somit die Empfindlichkeit, nicht übereinstimmen, dann müsste das Verhältnis der Brückenwiderstände anders gewählt werden.

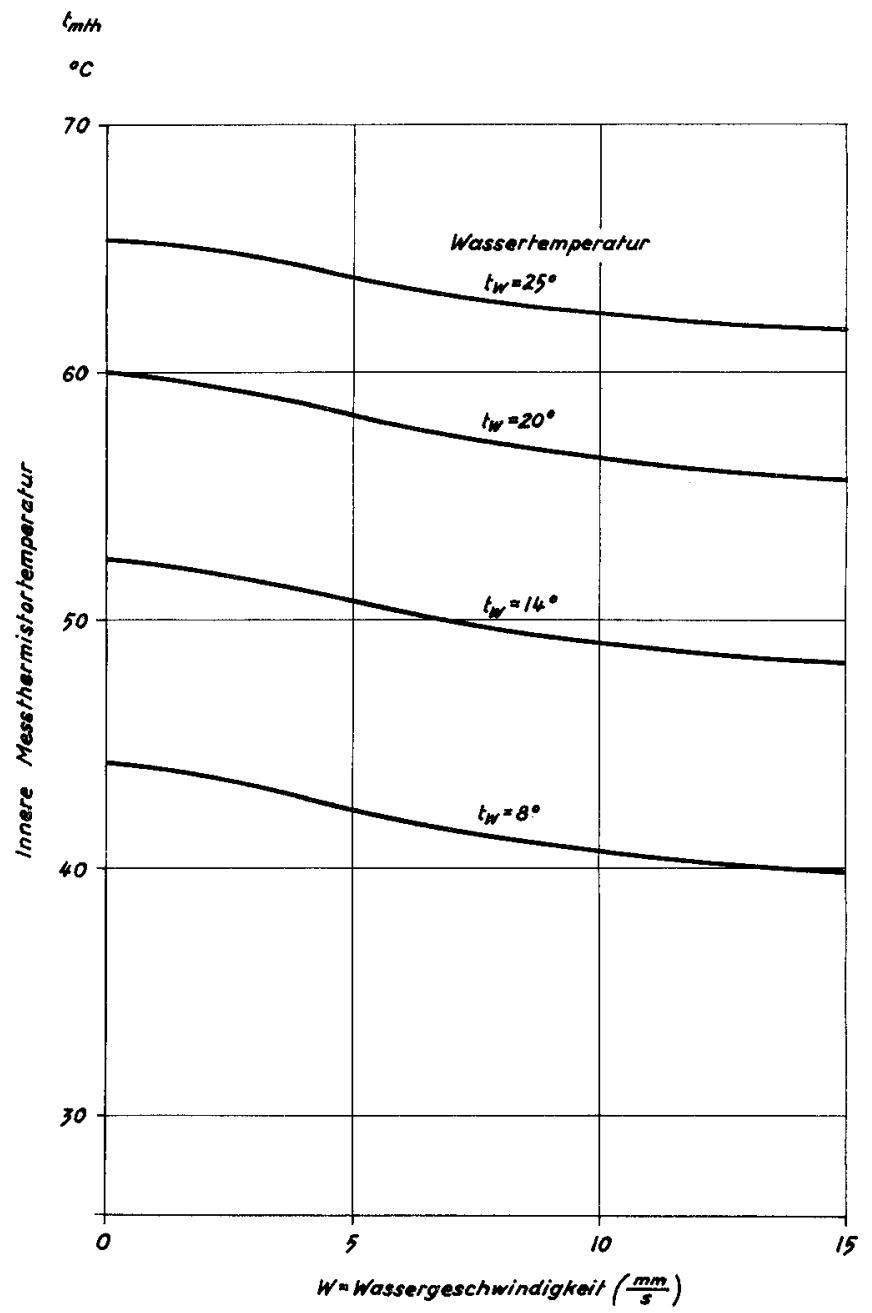

Abbildung 10

Temperatur des Messthermistors und Wassergeschwindigkeit. 


\subsection{Geschwindigkeitseichkurve}

Die Geschwindigkeitseichkurve (Abb. 9) zeigt den Instrumentenstrom $i$ in Funktion der Fliessgeschwindigkeit $\rightsquigarrow$. Das Mikroamperemeter hat einen Ausschlag von $50 \mu \mathrm{A}$ im Messbereich 1 und einen solchen von $150 \mu \mathrm{A}$ im Messbereich 2. Die Eichkurve hat somit einen empfindlichsten Geschwindigkeitsbereich. Darunter und darüber nimmt die Empfindlichkeit ab (Abb. 9).

Aus der Eichkurve kann mit Hilfe der Abb. 8 die Kurve $\frac{t_{m l h}-t_{w}}{Q}$ ermittelt werden. Mit dieser erhält man das aufschlussreiche, in Abb. 10 dargestellte Diagramm über die innere Messthermistortemperatur $t_{m t h}$ in Funktion der Geschwindigkeit $w$ und der Wassertemperatur $t_{w}$. Daraus ist ersichtlich, dass eine Geschwindigkeit von etwa $8 \mathrm{~mm} / \mathrm{s}$ eine Abkühlung von etwa $2^{\circ} \mathrm{C}$ verursacht (Abb. 10).

\section{B. Ausgeführte Messungen in Kläranlagen}

\section{Allgemeines}

Das wichtigste Anwendungsgebiet der Thermosonde sind Messungen in Klärbekken, da hier die meisten anderen Methoden der Strömungsmessung versagen oder aufwendig sind. Eine sehr aufschlussreiche Methode ist heute die Konzentrationsmessung mit radioaktiven Isotopen (GROcHE [4]). Leider ergeben auch diese Methoden nur Gesamtresultate über die Güte eines Beckens oder Beckenabschnitts, die allerdings bei dem Vergleich verschiedener Ausführungen sehr wertvoll sind. Um aber die Vorgänge festhalten und eventuelle Verbesserungen in strömungstechnischer Hinsicht erzielen zu können, muss man das Strömungsbild in den Becken kennen.

Das Messgerät mit der Thermosonde wurde speziell für Strömungsmessungen in Klärbecken entwickelt. Durch Verlängerung der Meßstange kann es bis zu $5 \mathrm{~m}$ Wassertiefe verwendet werden. Das Instrument wird mittels eines schwenkbaren Auslegers an der Räumerbrücke oder an einem Fussrost befestigt. Allerdings muss die Unterlage stabil sein, weil sonst durch die Strömung oder bei Betreten der Brücke Schwingungen angeregt werden, die das Messergebnis verfälschen können.

\section{Vorklärbecken Küsnacht (Zürich)}

Die ersten Versuche an einem praktischen Beispiel wurden in der Kläranlage Küsnacht unternommen. Die rechteckigen Vorklärbecken dieser Anlage sind unterirdisch angelegt, was gerade bei den ersten Versuchen, namentlich bei Regenwetter, sehr nützlich war.

Die auf Schienen laufende Längsräumerbrücke diente hier als Basis für die Befestigung des Messgerätes.

Die Becken besitzen Geiger-Einläufe. Der Zufluss wird zunächst mit einer Verteilrinne verteilt. Nachher werden die Einzeleinläufe beschickt, die gegeneinander gerichtet sind, um eine Vernichtung der Strömungsenergie zu erreichen.

Wie gut diese Aufgabe erreicht wurde, zeigt Abb. 11. Daraus gehen die Strömungsverhältnisse im Vorklärbecken bei Trockenwetteranfall hervor. Praktisch traten keine Zirkulations- bzw. Rückwärtsströmungen auf. Die Geschwindigkeitsverteilung ist ziemlich ausgeglichen. Die Wassermasse bewegt sich in der ersten Beckenhälfte hauptsächlich unten, in der zweiten Hälfte mehr an der Beckenoberfläche. 

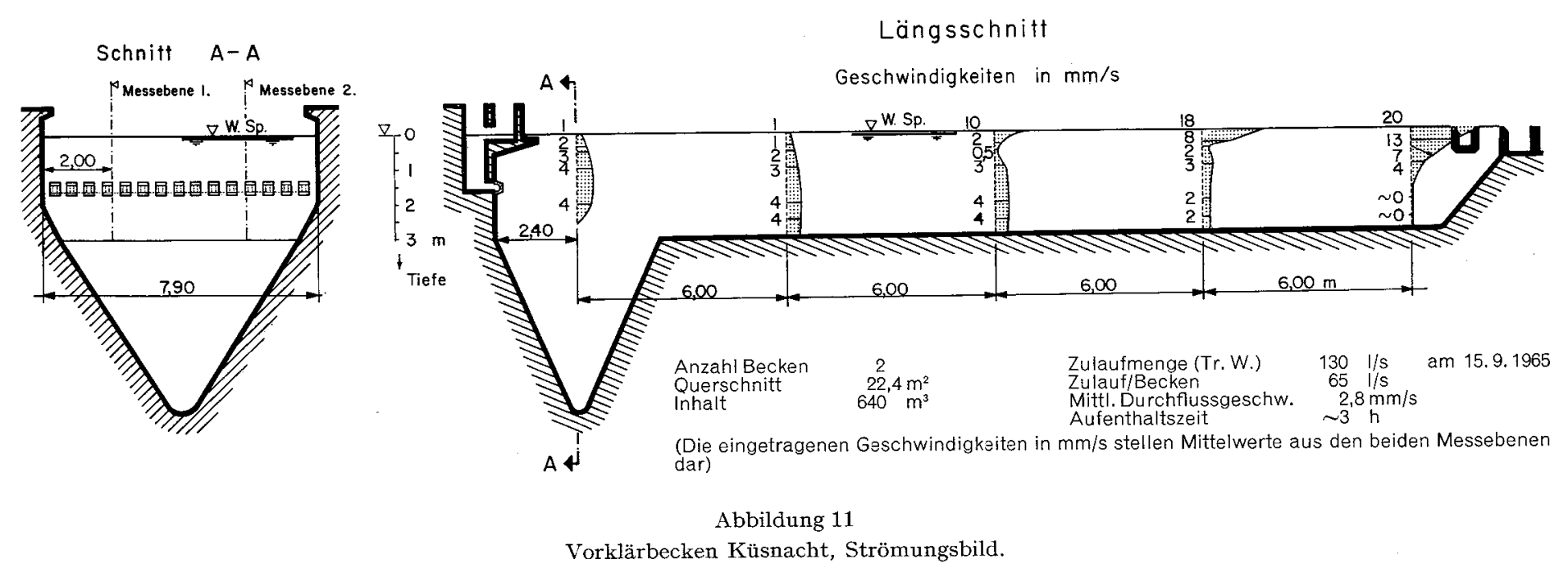

Vorklärbecken Küsnacht, Strömungsbild. 


\section{Nachklärbecken Küsnacht}

Dieses Becken ist, abgesehen vom andersgearteten Trichter, in den geometrischen Dimensionen ähnlich wie das Vorklärbecken. Auch das Einlaufsystem ist genau dasselbe, nur die Überläufe sind anders angeordnet. Die Anordnung zeigt Abb. 12.

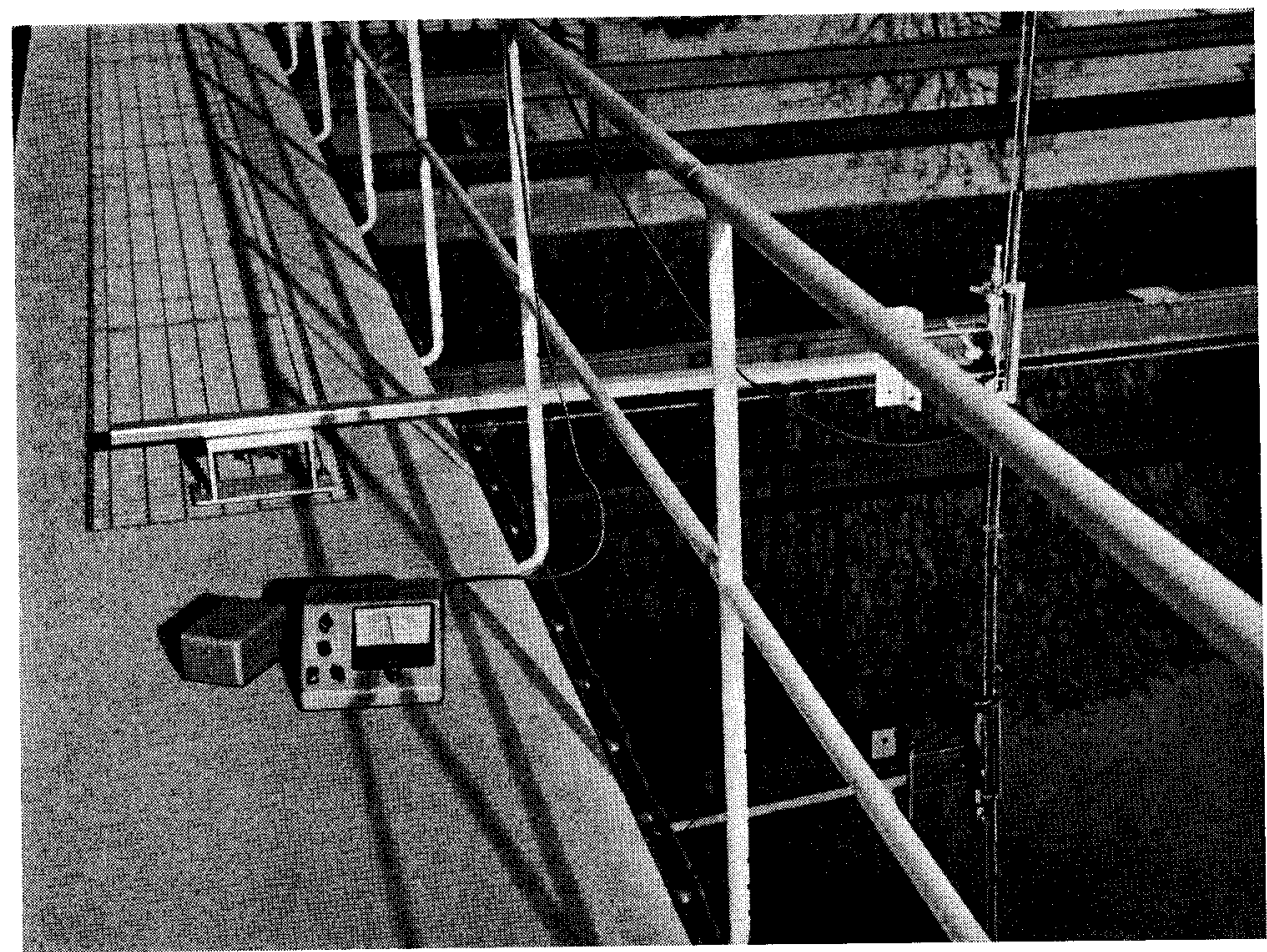

Abbildung 12

Nachklärbecken Küsnacht.

\subsection{Strömungsverhältnisse bei Trockenwetter}

Die Einlaufmenge des Wasserbelebtschlammgemisches ist ungefähr gleich wie beim Vorklärbecken. Trotzdem zeigt sich in Abb. 13 ein ganz anderes Strömungsbild. Die Strömung geht zuerst dem Boden entlang und kehrt nachher an einer bestimmten Stelle des Beckens um, wobei die hintere Hälfte desselben von dieser Zirkulation praktisch unberührt bleibt.

Die Ursache der starken Diskrepanz beider Strömungsbilder ist sehr wahrscheinlich im grösseren spezifischen Gewicht des zufliessenden Abwasserschlammgemisches zu suchen. Dieses Gemisch sinkt kurz nach dem Einlauf zu Boden und regt eine Zirkulation an.

Es ist klar, dass es schwierig ist, diesem Effekt entgegenzuwirken und eine gleichmässige Strömung zu erreichen. Der Abfluss der Anlage ist trotzdem klar, da die Nach-. klärbecken ausreichend dimensioniert sind. 

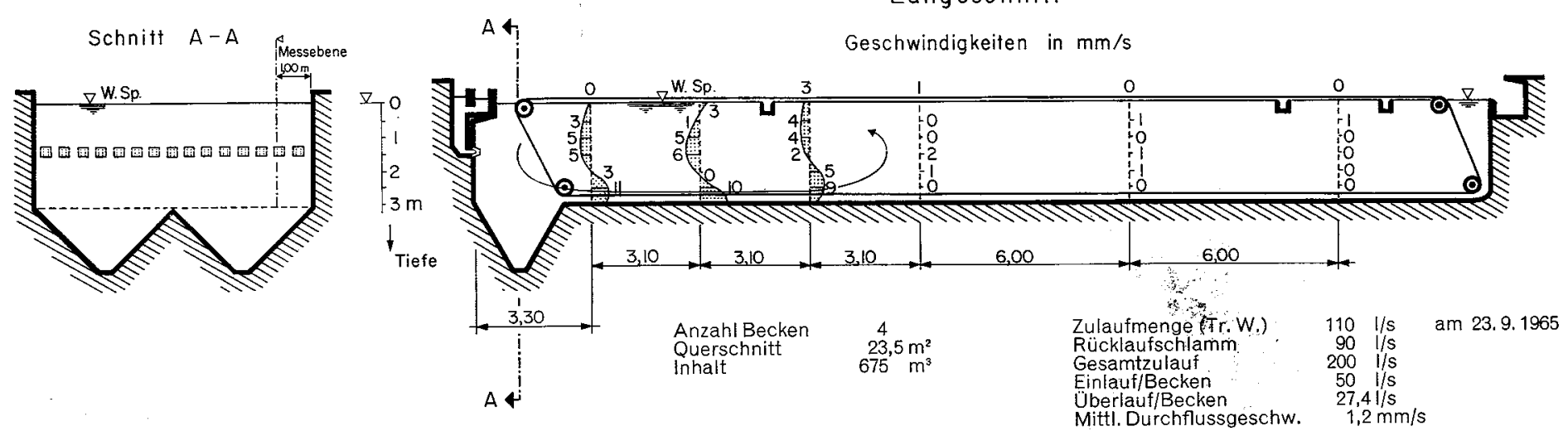

Abbildung 13. Nachklärbecken Küsnacht; Strömungsbild bei Trockenwetter.
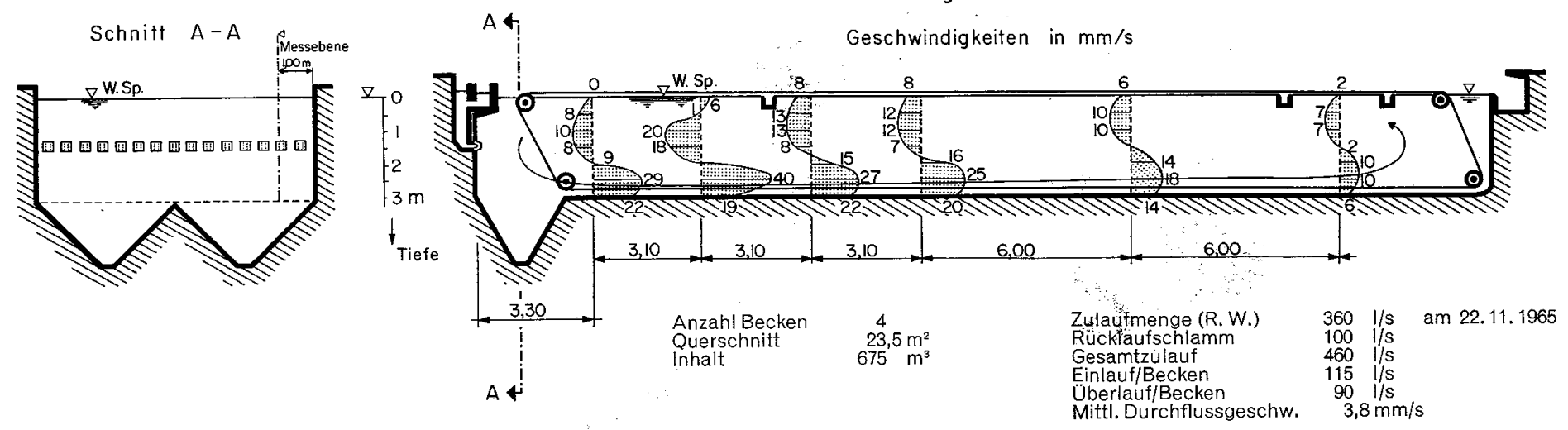


\subsection{Strömungsverhältnisse bei Regenwetter}

Wie Abb. 14 zeigt, ist hier die Zirkulation noch ausgeprägter als vorher. Sie reicht bis an das Ende des Beckens. Eine ruhende, von der Zirkulation unberührte Zone gibt es nicht mehr. Der Beckenabfluss ist bei stärkeren Regenfällen getrübt.

\section{Nachklärbecken Horgen (Zürich)}

Das Nachklärbecken ist als kreisrundes Flachbecken angelegt, wobei die Einlaufströmung durch ein zentrales Zylinderrohr nach unten gerichtet wird.

\subsection{Strömungsverhältnisse bei Trockenwetter}

Der grösste Teil der oberen Wasserschichten des Beckens ist praktisch zirkulationsfrei, wie Abb. 15 zeigt. Die Geschwindigkeiten liegen unterhalb der noch messbaren Werte. Nur etwa von 1,5 m Tiefe an bis zum Beckenboden ist eine Zirkulation vorhanden, wobei der Zulauf dem Boden entlang streicht

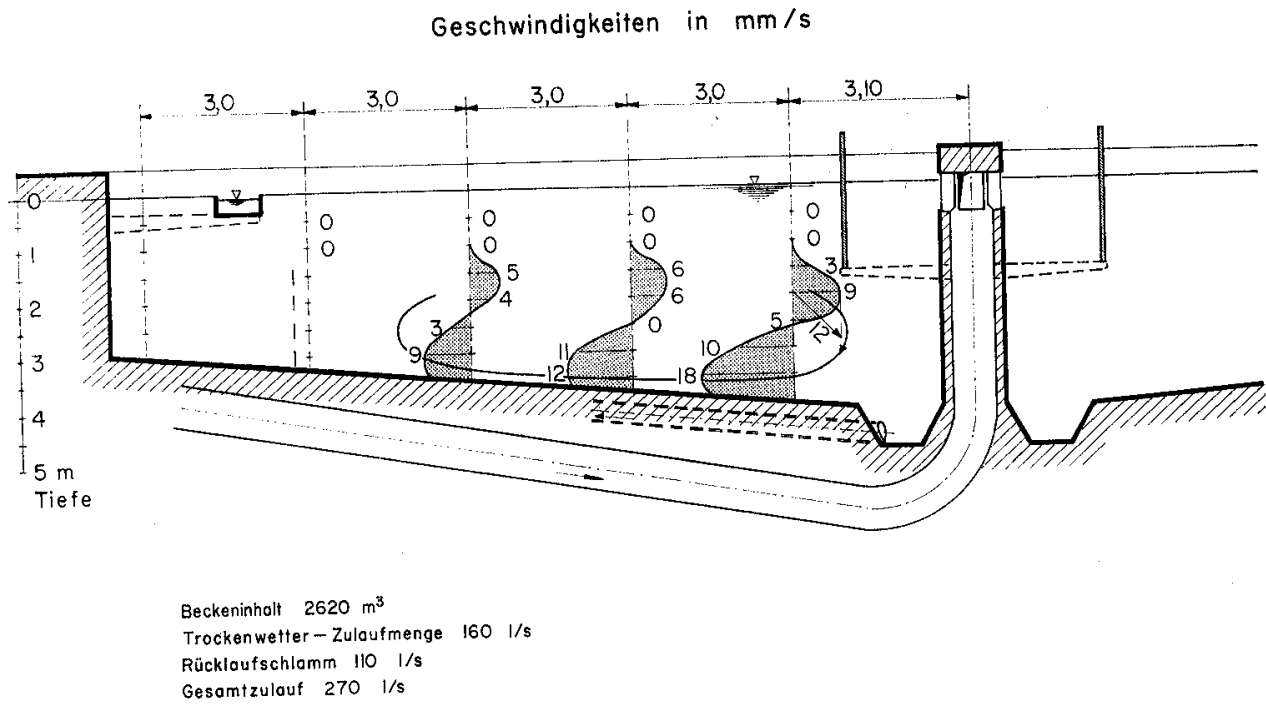

Abbildung 15

Nachklärbecken Horgen; Strömungsbild bei Trockenwetter.

\section{Belüftungsbecken Küsnacht (Zürich)}

Die Belüftung wird zurzeit durch eine Belüftungsbürste besorgt, wobei die Form des Beckenquerschnitts der Strömung angepasst ist (Abb. 16). An der Oberfläche und am Boden hat man starke eindeutige Geschwindigkeiten, die jede Schlammablagerung verhindern. In der mittleren Zone des Beckens war die Messung wegen der stossweisen, aus allen Richtungen kommenden Strömungen unbestimmt. 

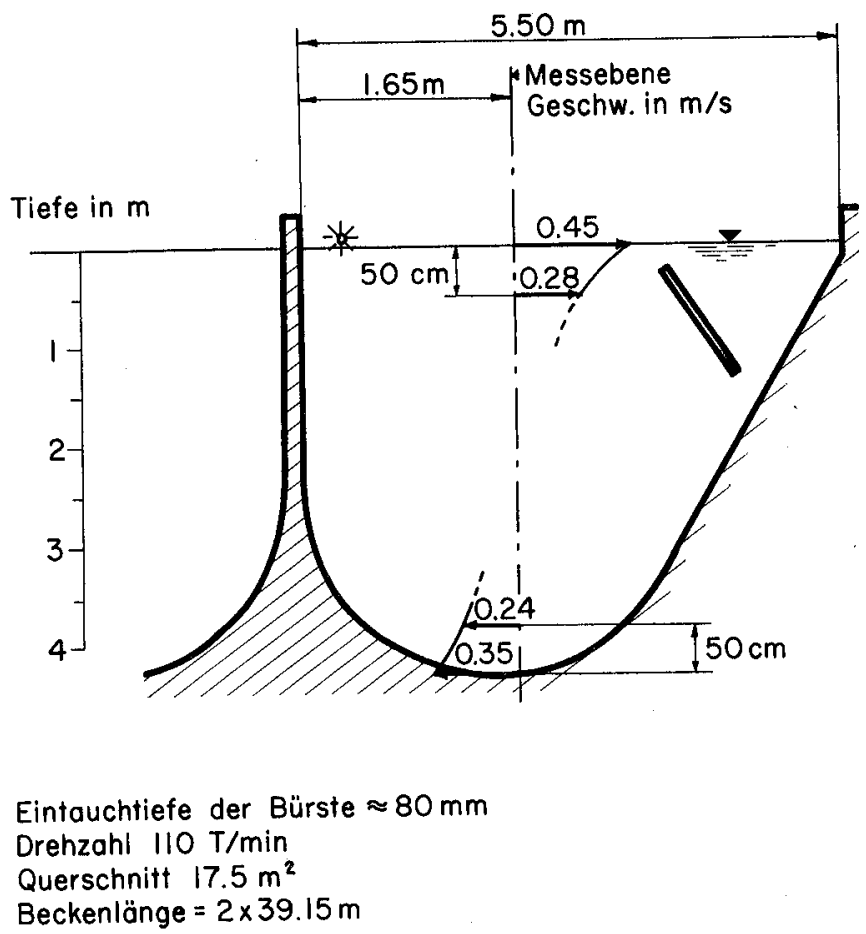

Abbildung 16

Belüftungsbecken Küsnacht: Strömungsbild.

\section{Zusammenfassung}

Strömungsmessungen mit Thermistoren sind relativ einfach und rasch durchführbar. Sie geben neue Erkenntnisse über die tatsächlichen Strömungsverhältnisse in den Becken. Sie sind geeignet, Unterlagen für die Verbesserung der Strömungsverhältnisse bei den heute üblichen Absetzbecken zu liefern und damit zur Erhöhung der Klärwirkung solcher Becken beizutragen.

\section{RÉSUMÉ}

Les mesures de courants au moyen de thermistors sont relativement simples et rapides. Elles procurent de nouvelles connaissances sur les conditions réelles des courants qui règnent dans les bassins de décantation. Elles contribuent ainsi à améliorer lesdites conditions dans les bassins actuels de décantation et, par conséquent, à augmenter l'effet de décantation de tels bassins.

\section{SUMMARY}

The measurement of water currents by means of thermistors is relatively simple and rapid. It gives new knowledge on the real conditions of the currents existing in sedimentation tanks. It helps to improve these conditions in the sedimentation basins used nowadays and contributes therefore to increase the sedimentation effect of such tanks. 


\section{LITERATURVERZEICHNIS}

[1] WÄHR, F., Das thermische Hydrometer. Wasserwirtschaft 50, 203 (1960).

[2] WACK, Die Messung kleiner Strömungsgeschwindigkeiten mit Thermosonde. Techn.Wiss. Mitt. Emschergenossensch. Nr. 6, 71 (1964).

[3] SchmidT-BREgas, Fr., Ưber die Ausbildung von vechteckigen Absetzbecken für häusliche Abwasser. Veröff. Inst. f. Siedlungswasserwirtsch. TH Hannover, H. 3, 22 (1958).

[4] Groche, D., Die Messung von Fliessvorgängen in ausgeführten Bawwerken der Abwasserveinigung mit Hilfe von künstlichen radioaktiven Isotopen und ihve Auswertung sowie Rückschlïsse auf die Konstruktionselemente. Stuttgarter Ber. Inst. f. Siedlungswasserwirtsch. Nr. 13 (1965).

[5] Standard Telephone \& Cables Ltd., Footsway, Kent (England), The Use and Application of Thermistors (1960).

[6] Philips, Eindhoven, Properties of NTC Resistors (1962).

Separatdruck aus der Schweiz. Zeitschrift für Hydrologie 28, Fasc. 1 (1966) Herausgegeben mit Unterstützung der Stiftung der Wirtschaft zur Förderung des Gewässerschutzes in der Schweiz 